\title{
Vorrede zur ersten Auflage.
}

Seit dem Erscheinen von Eduard Zellers Grundriß der Geschichte der griechischen Philosophie $\left(188_{3}\right)^{1}$ ist das Bedürfnis nach einer entsprechend knappen und zu Lehrzwecken verwendbaren Darstellung der Geschichte der neueren Philosophie noch fühlbarer geworden. Ein Seitenstück zu dem Kompendium des gefeierten Gelehrten, das die Resultate seines hochgeschătzten sechsbăndigen Werkes über die Philosophie der Griechen mit schlichter Klarheit zusammenfaßt, geben zu wollen, wäre ein vermessenes Beginnen gewesen; nur nach seiten praktischer Brauchbarkeit und sorgfaltiger Berücksichtigung der Bedürfnisse der Lernenden, über welche wir in regelmäßig an hiesiger Universităt abgehaltenen Repetitorien uns genauer zu informieren Gelegenheit hatten, durften wir hoffen, dem Vorbilde nicht allzufern zu bleiben.

Der vorliegende Grundriß soll zur Einführung, zur Repetition und zum Ersatze für Diktate bei akademischen Vorlesungen, desgleichen zur Orientierung für den weiteren Kreis der Gebildeten dienen. Dieser vorwiegend praktische Zweck des Buches gebot Zurückhaltung in der Geltendmachung persönlicher Überzeugungen und Einschrănkung der beurteilenden Reflexion zugunsten objektiver Darstellung; nur gelegentlich wurde ein kritischer Wink gegeben. Bei den minder bedeutenden Erscheinungen war die Oratio obliqua des Referates nicht zu vermeiden; aber wo es irgend anging, haben wir die Philosophen selbst ihre Lehren und Gründe entwickeln lassen, nicht sowohl in wörtlichen Auszügen aus ihren Schriften, als in freier verdichtender Reproduktion der Grundgedanken. Wenn die in der Einleitung und dem Rückblick am Schluß

1 Achte Aufage 1907 . 
ausgesprochene prinzipielle Auffassung von den die Philosophiegeschichte lenkenden Mächten und dem Entwickelungsgange der neueren Philosophie nicht überall an den historischen Tatsachen zur Einzelbewăhrung gebracht worden ist, so erklärt sich das aus der Rücksichtnahme teils auf den Umfang, teils auf den instruktiven Zweck des Buches. So wurde insbesondere der „psychologischen“ Erklärung der Systeme, als der eingånglicheren, aus pådagogischen Gründen ein breiterer Raum gegönnt, als nach unserer Meinung ihrer Bedeutung und Berechtigung entspricht. Mit Auswahl und Ausführlichkeit der Behandlung es jedem recht $\mathrm{zu}$ machen, ist unmöglich; möchten es nicht zu viele sein, die sich einen Leitfaden dieser Art ganz anders gedacht hătten. In der Gruppierung der Richtungen und Schulen und der Anordnung des Inhalts der Systeme um jeden Preis von den vorhandenen Darstellungen abzuweichen, lag nicht in unserer Absicht, ebensowenig, die Vorteile ungenutzt zu lassen, welche dem Späterkornmenden daraus erwachsen, daß ihm die hervorragenden Leistungen früherer Bearbeiter vorliegen. Insbesondere bekenuen wir dankbar die Förderung, welche uns die erneute Beschăftigung mit den einschlägigen Werken von Kuno Fischer, J. Ed. Erdmann, Zeller, Windelband, Überweg-Heinze, Harms, Lange, Franz Vorländer, Jodl und Pünjer gebracht hat.

Was uns bewog, die vorliegende Arbeit in Angriff zu nehmen, war die Wahrnehmung, daß ein Lehrbuch der Geschichte der neueren Philosophie fehle, das, reichhaltiger, gründlicher und prăziser als die kleinen Abrisse von Schwegler und Genossen, etwa die Mitte hielte zwischen der eleganten, jedoch ausführlicheren Darstellung Windelbands und dem soliden, aber mit seiner Spaltung des Textes in Paragraphen und Noten und der Unterbrechung desselben durch seitenlange Aufzăhlungen von Büchertiteln etwas trockenen Grundriß Überwegs. Während der erstere auf Literaturangaben gănzlich verzichtet, der letztere ihrer, wenigstens für Unterrichtszwecke, gar zu viele bringt und J. B. Meyers Leitfaden (1882) sich überhaupt auf bio- und bibliographische Notizen beschrankt, haben wir, mit möglichster Schonung der fortlaufenden Darstellung, im Text oder in den Anmerkungen außer den Hauptwerken der Philosophen einige von den Schriften über dieselben angeführt. Die leitenden Gesichtspunkte bei der Auswahl der Literatur, daß nur die wertvolleren und die als Lektüre für den Studierenden geeigneten Arbeiten aufzunehmen, außerdem aber die neuesten Erscheinungen tunlichst zu berücksichtigen seien, werden kaum Tadel zu befürchten haben. $\mathrm{DaB}$ manche uns unbekannte Schrift der Erwăhnung würdig gewesen wăre, soll nicht bcstritten werden. 
Die auf Anregung der Verlagsbuchhandlung im Anhang beigefügte Erläuterung einer An\%ahl philosophischer Termini, welche sich fast gan\% auf fremdsprachliche Ausdrücke beschränkt und die Bezeichnungen für die durchgehenden Richtungen bevor\%ugt, wurde nạch Möglichkeit so eingerichtet, daß sie zugleich als Sachregister benutzt werden kann.

Jena, 23. Dezember I 885 .

\section{Vorwort zur zweiten Auflage.}

Von den Verănderungen und Zusătzen der neuen Auflage entfăllt die Mehrzahl auf das erste und die beiden letzten Kapitel; von der allgemeinen Haltung der Darstellung abzugehen, fand ich keine Veranlassung. Mit aufrichtigem Danke gedenke ich der Anregungen, die mir sowohl dic öffentlichen Besprechungen als auch privatim geăußerte Wünsche gewåhrten. Gelegentlich traf es sich, daß widersprechende Forderungen sich begegneten - so wurde auf der einen Seite Erweiterung, auf der anderen Kürzung der Abschnitte über den deutschen Idealismus, insbesonderc iber Hegel, befürwortet -; da war ich denn freilich außerstande, beiden $\% u$ entsprechen. Unter den Rezensionen war mir die von B. Erdmann im ersten Bande des Archivs für Geschichte der Philosophie, unter den brieflichen Verbesserungsvorschlägen die von H. HeuBler von besonderem Werte. $\mathrm{Da}$ fremde Augen gewöhnlich schärfer sehen, so wäre es mir sehr willkommen, wenn mein Wunsch, diesen Grundriß immer nützlicher zu gestalten, auch ferner durch Ratschläge aus dem Leserkreise unterstützt würde. Sie werden, falls dem Buche die Gunst der Lehrer und der Lernenden erhalten bleibt, gewissenhaft berücksichtigt werden.

Für diejenigen, welche über zu große Fülle des Stoffes klagen, bemerke ich, daß sich durch Überspringung von Kap. I, 5 (Abschnitt I-3), 6, 8, : I2, I5 und I6 leicht Abhilfe schaffen läbt.

Erlangen, Ir. Juni 1892. 